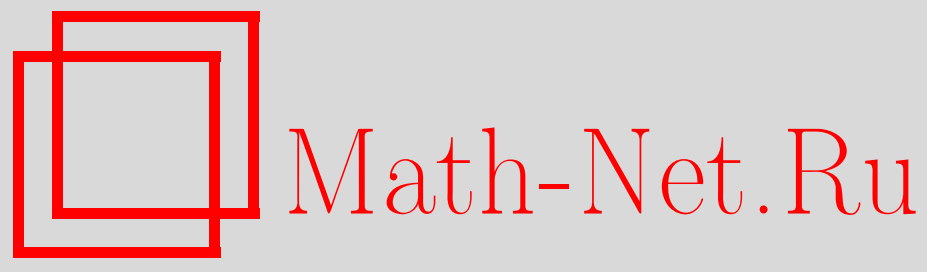

А. В. Колчин, В. Ф. Колчин, О переходе распределений сумм независимых одинаково распределенных случайных величин с одной решетки на другую в обобщенной схеме размещения, Дискрет. матем., 2006, том 18, выпуск 4, 113-127

DOI: https://doi.org/10.4213/dm76

Использование Общероссийского математического портала Math-Net.Ru подразумевает, что вы прочитали и согласны с пользовательским соглашением http://www . mathnet.ru/rus/agreement

Параметры загрузки:

IP : 18.207 .199 .55

26 апреля 2023 г., 07:20:11

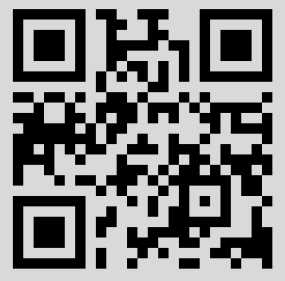




\title{
О переходе распределений сумм независимых одинаково распределенных случайных величин с одной решетки на другую в обобщенной схеме размещения
}

\author{
() 2006 г. А. В. Колчин, В. Ф. Колчин
}

\begin{abstract}
Рассматривается эффект перехода распределений сумм независимых одинаково распределенных неотрицательных целочисленных случайных величин с одной решетки на другую в контексте обобщенной схемы размещения.

Работа выполнена при поддержке Российского фонда фундаментальных исследований, проект 05-01-00035, программой Отделения математических наук РАН «Современные проблемы теоретической математики» и программой Президента Российской Федерации поддержки ведущих научных школ, грант НШ 4129.2006.1.
\end{abstract}

\section{1. Введение}

В вероятностной комбинаторике получил распространение подход, основанный на применении обобщенной схемы размещения, позволяющий сводить ряд комбинаторных задач к задачам о суммах независимых случайных величин, классическому объекту изучения в теории вероятностей [7-10, 14-20]. Обобщенная схема размещения была введена в [7] и заняла заметное место в асимптотических исследованиях в вероятностной комбинаторике. Свое название эта схема получила в связи с тем, что она является обобщением классической задачи о случайном размещении частиц по ячейкам [8].

В обобщенной схеме размещения частиц распределение заполнений ячеек представимо как условное распределение независимых случайных величин при условии, что их сумма принимает фиксированное значение. Пусть $\eta_{1}, \ldots, \eta_{N}$ - неотрицательные целочисленные случайные величины, рассматриваемые как некоторые числовые характеристики комбинаторной структуры из $N$ компонент, состоящей из $n$ элементов, такие, что $\eta_{1}+\ldots+\eta_{N}=n$. Если существуют независимые случайные величины $\xi_{1}, \ldots, \xi_{N}$ такие, что совместное распределение $\eta_{1}, \ldots, \eta_{N}$ допускает представление

$$
\mathbf{P}\left\{\eta_{1}=k_{1}, \ldots, \eta_{N}=k_{N}\right\}=\mathbf{P}\left\{\xi_{1}=k_{1}, \ldots, \xi_{N}=k_{N} \mid \xi_{1}+\ldots+\xi_{N}=n\right\},
$$

где $k_{1}, \ldots, k_{N}$ - произвольные целые числа, то говорят, что $\eta_{1}, \ldots, \eta_{N}$ образуют обобщенную схему размещения с параметрами $n$ и $N$ и независимыми случайными величинами $\xi_{1}, \ldots, \xi_{N}$. Случайные величины $\eta_{1}, \ldots, \eta_{N}$ интерпретируются как заполнения ячеек. 
В силу независимости случайных величин $\xi_{1}, \ldots, \xi_{N}$, изучение многих характеристик обобщенной схемы размещения сводится к задачам о суммах независимых случайных величин.

Например, пусть случайные величины $\xi_{1}, \ldots, \xi_{N}$ одинаково распределены и пусть $\mu_{l}$ - число случайных величин $\eta_{1}, \ldots, \eta_{N}$, принявших значение $l, l=0,1, \ldots, N$. Нетрудно показать (см., например, [8]), что

$$
\mathbf{P}\left\{\mu_{l}=k\right\}=\left(\begin{array}{l}
N \\
k
\end{array}\right) p_{l}^{k}\left(1-p_{l}\right)^{N-k} \frac{\mathbf{P}\left\{\xi_{1}^{(l)}+\ldots+\xi_{N-k}^{(l)}=n-k l\right\}}{\mathbf{P}\left\{\xi_{1}+\ldots+\xi_{N}=n\right\}},
$$

где $\xi_{1}^{(l)}, \ldots, \xi_{N-k}^{(l)}$ - независимые одинаково распределенные случайные величины,

$$
\mathbf{P}\left\{\xi_{1}^{(l)}=k\right\}=\mathbf{P}\left\{\xi_{1}=k \mid \xi_{1} \neq l\right\}, \quad k=0,1,2, \ldots,
$$

и

$$
p_{l}=\mathbf{P}\left\{\xi_{1}=l\right\}, \quad l=0,1,2, \ldots
$$

Таким образом, получение предельного распределения случайной величины $\mu_{l}$ сводится к применению локальных теорем для сумм независимых случайных величин. В случае, когда распределения слагаемых одинаковы и фиксированы (не зависят от числа слагаемых), можно пользоваться хорошо развитой теорией суммирования независимых случайных величин [1]. Однако во многих применениях обобщенной схемы возникает необходимость в локальных предельных теоремах в схеме серий. В таких случаях до сих пор нет исчерпывающего ответа на вопрос, справедлива ли локальная предельная теорема даже в случае сходимости к нормальному закону; для каждого конкретного случая приходится проводить отдельное доказательство, либо следуя доказательству локальной теоремы, предложенному Б. В. Гнеденко $[1,2,11,13,14]$, либо проверяя условия общих локальных теорем $[3,12]$.

Обычно (см., например, [9]), распределение случайных величин $\xi_{1}, \ldots, \xi_{N}$ представляется в виде

$$
\mathbf{P}\left\{\xi_{1}=k\right\}=\frac{b_{k} \theta^{k}}{k ! B(\theta)}
$$

где $b_{0}, b_{1}, b_{2}, \ldots$ - некоторая последовательность неотрицательных чисел,

$$
B(\theta)=\sum_{k=0}^{\infty} \frac{b_{k} \theta^{k}}{k !}
$$

и $\theta$ - параметр, принимающий положительные значение из области сходимости ряда $B(\theta)$. В таких случаях, если соотношение (1) справедливо при некотором значении $\theta$, то оно остается верным при всех положительных $\theta$ из области сходимости ряда $B(\theta)$ (см., например, $[8,9,10])$.

Для изучения характеристик обобщенной схемы размещения, как правило, требуются локальные предельные теоремы при всех значениях параметра $\theta$.

В [6] были рассмотрены простейшие случаи. Предполагалось, что $b_{0}$ и $b_{1}$ строго положительны и допускались лишь значения параметра $\theta$, не приближающиеся к значению радиуса сходимости ряда $B(\theta)$. При этих ограничениях были получены предельные теоремы о сходимости к нормальному распределению и распределению Пуассона. 
В настоящей статье рассматривается эффект перехода распределений сумм независимых одинаково распределенных целочисленных неотрицательных случайных величин с распределением вида (2) с одной решетки на другую. В схеме размещения частищ по ячейкам этот эффект был описан в [8] для распределения числа ячеек, содержащих ровно одну частицу.

В случае, когда значения параметра $\theta$ приближаются к границе сходимости ряда $B(\theta)$, как показано на примерах в $[4,5,9,10])$, могут появляться и другие предельные распределения.

Некоторые из доказанных в [6] и настоящей статье теорем, по-видимому, могут быть получены путем проверки приведенных в [12] общих условий справедливости локальных теорем о сходимости к нормальному распределению (см. [3]), однако авторы считают, что наличие простых прямых доказательств может оказаться полезным для дальнейших исследований в этой области.

\section{2. Основные обозначения. Вспомогательные соотношения}

Мы будем придерживаться следующих обозначений. Пусть задана последовательность неотрицательных чисел $b_{0}, b_{1}, b_{2}, \ldots$, таких, что радиус сходимости $R$ ряда

$$
B(\theta)=\sum_{k=0}^{\infty} \frac{b_{k} \theta^{k}}{k !}
$$

положителен. Введем целочисленную случайную величину $\xi=\xi(\theta)$, распределенную по следующему закону:

$$
\mathbf{P}\{\xi=k\}=\frac{b_{k} \theta^{k}}{k ! B(\theta)}, \quad k=0,1,2, \ldots
$$

Будем говорить, что выполнено условие $A_{r}, r \geqslant 2$, если $b_{0}>0, b_{1}=\ldots=b_{r-1}=0$, $b_{r}>0$, и максимальный шаг распределения (3) равен единище. Если $b_{0}, b_{1}>0$, то будем говорить, что выполнено условие $A_{1}$.

Положим

$$
m=m(\theta)=\mathbf{M} \xi, \quad \sigma^{2}=\sigma^{2}(\theta)=\mathbf{D} \xi, \quad \beta=\beta(\theta)=\mathbf{M}|\xi-m|^{3} .
$$

Заметим, что при $|\theta|<R$ случайная величина $\xi$ имеет все моменты.

Пусть $\xi_{1}, \xi_{2}, \ldots$ - независимые случайные величины, распределение которых совпадает с распределением (3) случайной величины $\xi$.

Нашей целью является получение предельных теорем для сумм вида

$$
S_{N}=\sum_{k=1}^{N} \xi_{k}
$$

при $N \rightarrow \infty$ и различных вариантах поведения параметра $\theta=\theta(N)$. Положим

$$
P_{N}(n)=\mathbf{P}\left\{S_{N}=n\right\} \text {. }
$$


Обозначим через $F_{N}(x)$ функцию распределения суммы центрированных и нормированных случайных величин

$$
S_{N}^{*}=\sum_{k=1}^{N} \frac{\xi_{k}-m}{\sigma \sqrt{N}}
$$

Легко видеть, что производящая функция распределения случайной величины $\xi$ есть

$$
\phi(z)=\sum_{k=0}^{\infty} \frac{b_{k} \theta^{k} z^{k}}{k ! B(\theta)}=\frac{1}{B(\theta)} \sum_{k=0}^{\infty} \frac{b_{k}(z \theta)^{k}}{k !}=\frac{B(z \theta)}{B(\theta)}
$$

Характеристическая функщия $\varphi(t)$ случайной величины $\xi$ есть $\phi\left(e^{i t}\right)$, поэтому

$$
\varphi(t)=\frac{B\left(\theta e^{i t}\right)}{B(\theta)}
$$

Далее, характеристическая функция $\varphi^{*}(t)$ центрированной и нормированной случайной величины $(\xi-m) /(\sigma \sqrt{N})$ есть $\phi\left(e^{i t /(\sigma \sqrt{N})}\right) e^{-i t m /(\sigma \sqrt{N})}$, поэтому

$$
\varphi^{*}(t)=\frac{B\left(\theta e^{i t /(\sigma \sqrt{N})}\right)}{B(\theta)} e^{-i t m /(\sigma \sqrt{N})} .
$$

Через $\bar{\varphi}(t)=$ М $e^{i t(\xi-m)}$ обозначим характеристическую функцию центрированной случайной величины $\xi-m$.

Легко видеть, что первые два момента случайной величины $\xi$ выражаются через функцию $B(\theta)$ следующим образом:

$$
\begin{aligned}
m=\mathbf{M} \xi & =\sum_{k=1}^{\infty} \frac{k b_{k} \theta^{k}}{k ! B(\theta)}=\frac{\theta B^{\prime}(\theta)}{B(\theta)} \\
\mathbf{M} \xi(\xi-1) & =\sum_{k=2}^{\infty} \frac{k(k-1) b_{k} \theta^{k}}{k ! B(\theta)}=\frac{\theta^{2} B^{\prime \prime}(\theta)}{B(\theta)} \\
\sigma^{2}=\mathbf{D} \xi & =\mathbf{M} \xi(\xi-1)+\mathbf{M} \xi-(\mathbf{M} \xi)^{2} \\
& =\frac{\theta^{2} B^{\prime \prime}(\theta)}{B(\theta)}+\frac{\theta B^{\prime}(\theta)}{B(\theta)}-\frac{\theta^{2}\left(B^{\prime}(\theta)\right)^{2}}{(B(\theta))^{2}}
\end{aligned}
$$

\section{3. Предельные теоремы}

\section{1. Интегральные предельные теоремы}

В [6] предполагалось выполнение условия $A_{1}$. Оказывается, что в доказательстве интегральной предельной теоремы о слабой сходимости распределений сумм $S_{N}^{*}$ к стандартному нормальному закону не требуется выполнения каких-либо арифметических ограничений на последовательность $b_{0}, b_{1}, \ldots$ 
Теорема 1. Пусть $N \rightarrow \infty$ и существуют постоянные $\theta_{0}, \theta_{1}, 0<\theta_{0}<\theta_{1}<R$, такие, что параметр $\theta=\theta(N) \in\left[\theta_{0}, \theta_{1}\right]$. Тогда распределение $S_{N}^{*}$ слабо сходится к стандартному нормальному закону, причем справедлива оченка

$$
\sup _{x}\left|F_{N}(x)-\Phi(x)\right| \leqslant c \frac{\beta^{*}}{\left(\sigma^{*}\right)^{3} \sqrt{N}},
$$

где $\Phi(x)$ - функция распределения стандартного нормального закона и

$$
\sigma^{*}=\min _{\theta_{0} \leqslant \theta \leqslant \theta_{1}} \sigma(\theta)>0, \quad \beta^{*}=\max _{\theta_{0} \leqslant \theta \leqslant \theta_{1}} \beta(\theta),
$$

с - постоянная из неравенства Берри-Эссеена.

Доказательство. Справедливость теоремы следует из того, что если зафиксировать произвольное значение параметра $\theta$, то по теореме Берри-Эссеена

$$
\sup _{x}\left|F_{N}(x)-\Phi(x)\right| \leqslant c \frac{\beta(\theta)}{(\sigma(\theta))^{3} \sqrt{N}} \leqslant c \frac{\beta^{*}}{\left(\sigma^{*}\right)^{3} \sqrt{N}}
$$

положительность $\sigma^{*}$ следует из того, что $\sigma(\theta)$ есть непрерывная функция от $\theta$ и $\sigma(\theta)>0$ при $\theta>0$ при выполнении любого из условий $A_{r}, r \geqslant 1$.

Теорема 2. Пусть выполнено условие $A_{r}, r \geqslant 1$. Пусть $N \rightarrow \infty$, и пусть параметр $\theta=\theta(N) \rightarrow 0$ так, что выполняется соотношение

$$
\frac{b_{r}}{r ! b_{0}} N \theta^{r} \rightarrow \lambda
$$

где $\lambda$ - некоторая положительная постоянная. Тогда распределение суммы $S_{N} / r$ слабо сходится к распределению Пуассона с параметром $\lambda$.

Доказательство. Применим теорему непрерывности для производящих функщий (см., например, [11]). Производящая функция случайной величины, имеющей распределение Пуассона с параметром $\lambda$ на решетке целых неотрицательных чисел, кратных $r$, равна

$$
\phi_{\Pi}^{(r)}(z)=\sum_{k=0}^{\infty} \frac{\lambda^{k} e^{-\lambda}}{k !} z^{k r}=e^{-\lambda\left(1-z^{r}\right)} .
$$

Докажем, что при выполнении условий теоремы производящая функция $\phi^{N}(z)$ случайной величины $S_{N}$ стремится к $\phi_{\Pi}^{(r)}(z)$ при любом фиксированном $z, 0<z<1$. Пользуясь асимптотическим разложением

$$
\ln (1+x)=x+O\left(x^{2}\right)
$$

справедливым при $|x|<1$, получаем, что при выполнении условий теоремы

$$
\begin{aligned}
\ln \phi^{N}(z) & =N(\ln B(\theta z)-\ln B(\theta)) \\
& =N \ln \left(1+\frac{b_{r}(\theta z)^{r}}{b_{0} r !}+O\left(\theta^{r+1}\right)\right)-N \ln \left(1+\frac{b_{r} \theta^{r}}{b_{0} r !}+O\left(\theta^{r+1}\right)\right) \\
& =\frac{b_{r}}{b_{0}} \frac{\theta^{r}}{r !} N\left(z^{r}-1\right)+O\left(N \theta^{r+1}\right)=\lambda\left(z^{r}-1\right)(1+o(1)),
\end{aligned}
$$

что завершает доказательство теоремы. 
Теорема 3. Пусть выполнено условие $A_{r}, r \geqslant 1$. Пусть $N \rightarrow \infty$ и пусть параметр $\theta=\theta(N) \rightarrow 0$ так, что $\theta^{r} N \rightarrow \infty$. Тогда распределение суммы $S_{N}^{*}$ слабо сходится $\kappa$ стандартному нормальному закону.

Доказательство. Случай $r=1$ рассмотрен в [6]. Поэтому предполагаем, что $r \geqslant 2$. Покажем, что при условиях теоремы $\left(\varphi^{*}(t)\right)^{N} \rightarrow e^{-t^{2} / 2}$.

Представим $B\left(\theta e^{i t /(\sigma \sqrt{N})}\right)$ в виде

$$
B\left(\theta e^{i t /(\sigma \sqrt{N})}\right)=B(\theta+\Delta \theta), \quad \Delta=e^{i t /(\sigma \sqrt{N})}-1 .
$$

Возьмем логарифм от обеих частей представления характеристической функщии (4). Разложим $\ln B(\theta+\Delta \theta)$ в точке $\theta$ при фиксированном $t$ до членов третьего порядка малости, но вначале выясним асимптотическое поведение производных функщии $B(\theta)$ в точке $\theta$. Нетрудно видеть, что при выполнении условия $A_{r}, r \geqslant 2$,

$$
\begin{aligned}
B(\theta) & =b_{0}+\frac{b_{r} \theta^{r}}{r !}+O\left(\theta^{r+1}\right), \\
B^{\prime}(\theta) & =\frac{b_{r} \theta^{r-1}}{(r-1) !}+O\left(\theta^{r}\right), \\
B^{\prime \prime}(\theta) & =\frac{b_{r} \theta^{r-2}}{(r-2) !}+O\left(\theta^{r-1}\right),
\end{aligned}
$$

и

$$
\left|B^{\prime \prime \prime}(\theta)\right|= \begin{cases}O(1), & r=2, \\ O\left(\theta^{r-3}\right), & r \geqslant 3 .\end{cases}
$$

Поэтому при фиксированном $t$

$$
\ln B(\theta+\Delta \theta)=\ln \left(B(\theta)+\Delta \theta B^{\prime}(\theta)+\frac{(\Delta \theta)^{2}}{2} B^{\prime \prime}(\theta)+R_{r}\right),
$$

где $R_{r}=R_{r}(\Delta, \theta)=O\left(\Delta^{3} \theta^{3}\left|B^{\prime \prime \prime}(\theta)\right|\right)$, так что $R_{2}=R_{2}(\Delta, \theta)=O\left(\Delta^{3} \theta^{3}\right)$ и $R_{r}=R_{r}(\Delta, \theta)=O\left(\Delta^{3} \theta^{r}\right), r \geqslant 3$. Далее, пользуясь разложением

$$
\ln (1+x)=x-\frac{x^{2}}{2}+O\left(x^{3}\right)
$$

при $x \rightarrow 0$, с учетом соотношения (6) получаем, что

$$
\begin{aligned}
\ln B(\theta+\Delta \theta) & =\ln B(\theta)+\ln \left(1+\frac{\Delta \theta B^{\prime}(\theta)}{B(\theta)}+\frac{\Delta^{2} \theta^{2} B^{\prime \prime}(\theta)}{2 B(\theta)}+\frac{R_{r}}{B(\theta)}\right) \\
& =\ln B(\theta)+\frac{\Delta \theta B^{\prime}(\theta)}{B(\theta)}+\frac{\Delta^{2} \theta^{2} B^{\prime \prime}(\theta)}{2 B(\theta)}-\frac{\Delta^{2} \theta^{2}\left(B^{\prime}(\theta)\right)^{2}}{2(B(\theta))^{2}}+O\left(R_{r}\right) .
\end{aligned}
$$

Отсюда получаем, что

$$
\begin{aligned}
\ln \varphi^{*}(t) & =\ln \frac{B\left(\theta e^{i t /(\sigma \sqrt{N})}\right)}{B(\theta)} e^{-i t m /(\sigma \sqrt{N})} \\
& =\ln B(\theta+\Delta \theta)-\ln B(\theta)-i t m /(\sigma \sqrt{N}) \\
& =\frac{\Delta \theta B^{\prime}(\theta)}{B(\theta)}+\frac{\Delta^{2} \theta^{2} B^{\prime \prime}(\theta)}{2 B(\theta)}-\frac{\Delta^{2} \theta^{2}\left(B^{\prime}(\theta)\right)^{2}}{2(B(\theta))^{2}}-\frac{i t m}{\sigma \sqrt{N}}+O\left(R_{r}\right)
\end{aligned}
$$


Легко видеть, что при $\theta \rightarrow 0$, в силу ранее полученных представлений для $m(\theta)$ и $\sigma^{2}(\theta)$ и асимптотических выражений для производных функции $B(\theta)$, справедливы представления

$$
\begin{aligned}
m & =m(\theta)=\mathbf{M} \xi=\theta \frac{B^{\prime}(\theta)}{B(\theta)}=\frac{b_{r} \theta^{r}}{b_{0}(r-1) !}+O\left(\theta^{r+1}\right), \\
\sigma^{2} & =\sigma^{2}(\theta)=\mathbf{D} \xi=\frac{\theta^{2} B^{\prime \prime}(\theta)}{B(\theta)}+\frac{\theta B^{\prime}(\theta)}{B(\theta)}-\frac{\theta^{2}\left(B^{\prime}(\theta)\right)^{2}}{(B(\theta))^{2}} \\
& =\frac{b_{r} \theta^{r}}{b_{0}(r-2) !}+\frac{b_{r} \theta^{r}}{b_{0}(r-1) !}+O\left(\theta^{r+1}\right)=\frac{r b_{r} \theta^{r}}{b_{0}(r-1) !}+O\left(\theta^{r+1}\right) .
\end{aligned}
$$

Далее, очевидно, что

$$
\Delta \frac{\theta B^{\prime}(\theta)}{B(\theta)}-\frac{i t m}{\sigma \sqrt{N}}=\Delta m-\frac{i t m}{\sigma \sqrt{N}} .
$$

При $N \rightarrow \infty$ при любом фиксированном $t$

$$
\Delta=e^{i t /(\sigma \sqrt{N})}-1=\frac{i t}{\sigma \sqrt{N}}-\frac{t^{2}}{2 \sigma^{2} N}+O\left(\frac{1}{\sigma^{3} N^{3 / 2}}\right)
$$

откуда следует, что

$$
\Delta m-\frac{i t m}{\sigma \sqrt{N}}=-\frac{t^{2} m}{2 \sigma^{2} N}+O\left(\frac{1}{\theta^{r / 2} N^{3 / 2}}\right)
$$

поскольку в условиях теоремы

$$
\sigma^{2} N=\frac{r b_{r} N \theta^{r}}{b_{0}(r-1) !}+O\left(N \theta^{r+1}\right) \rightarrow \infty
$$

здесь используется условие, что $\theta^{r} N \rightarrow \infty$.

Окончательно получаем следующую оценку для логарифма характеристической функции случайной величины $\xi$ :

$$
\begin{aligned}
\ln \varphi^{*}(t) & =-\frac{t^{2} m}{2 \sigma^{2} N}+\frac{\Delta^{2}}{2}\left(\frac{\theta^{2} B^{\prime \prime}(\theta)}{B(\theta)}-\frac{\left(\theta B^{\prime}(\theta)\right)^{2}}{(B(\theta))^{2}}\right)+O\left(R_{r}\right) \\
& =-\frac{t^{2} m}{2 \sigma^{2} N}-\frac{t^{2}}{2 \sigma^{2} N}\left(\mathbf{M} \xi(\xi-1)-(\mathbf{M} \xi)^{2}\right)+O\left(R_{r}\right) \\
& =-\frac{t^{2}}{2 \sigma^{2} N}\left(\mathbf{M} \xi+\mathbf{M} \xi(\xi-1)-(\mathbf{M} \xi)^{2}\right)+O\left(R_{r}\right)=-\frac{t^{2}}{2 N}+O\left(R_{r}\right),
\end{aligned}
$$

где

$$
R_{2}=O\left(\Delta^{3} \theta^{3}\right)=O\left(\frac{1}{N^{3 / 2}}\right), \quad R_{r}=O\left(\Delta^{3} \theta^{r}\right)=O\left(\frac{1}{\theta^{r / 2} N^{3 / 2}}\right), \quad r \geqslant 3
$$

Поэтому

$$
\left(\varphi^{*}(t)\right)^{N}= \begin{cases}e^{-t^{2} / 2}+O(1 / \sqrt{N}), & r=2 \\ e^{-t^{2} / 2}+O\left(1 / \sqrt{\theta^{r} N}\right), & r \geqslant 3\end{cases}
$$

что завершает доказательство теоремы. 


\section{2. Локальные предельные теоремы}

Введем дополнительные обозначения. Пусть выполнено условие $A_{r}$ при некотором $r \geqslant 2$, и пусть $s$ - первое большее $r$ число, для которого $b_{s}>0$. Положим

$$
z=\frac{n-N m}{\sigma \sqrt{N}}
$$

где, как и ранее, $m=M \xi, \sigma^{2}=D \xi$.

Теорема 4. Пусть выполнено условие $A_{r}$ при некотором $r \geqslant 2, N \rightarrow \infty, \theta=\theta(N) \rightarrow 0$ так, что $N \theta^{s} \rightarrow \infty$ и максимальный шаг решетки, на которой лежат точки $0, r$ u $s$, равен единиче. Тогда

$$
\sigma \sqrt{N} P_{N}(n)-\frac{1}{\sqrt{2 \pi}} e^{-z^{2} / 2} \rightarrow 0
$$

равномерно относительно чельх неотричательных $n$.

Доказательство. Напомним, что характеристическая функщия случайной величины $\xi$ равна $\varphi(t)=B\left(\theta e^{i t}\right) / B(\theta), \bar{\varphi}(t)=\varphi(t) e^{-i t m}-$ характеристическая функция центрированной случайной величины $\xi-m$ и $\varphi^{*}(t)=\bar{\varphi}(t /(\sigma \sqrt{n}))=\varphi(t /(\sigma \sqrt{n})) e^{-i t m /(\sigma \sqrt{n})}$ - характеристическая функция центрированной и нормированной случайной величины $(\xi-m) /(\sigma \sqrt{N})$.

По теореме обращения для характеристических функций

$$
P_{N}(n)=\frac{1}{2 \pi} \int_{-\pi}^{\pi} \varphi^{N}(t) e^{-i t n} d t
$$

Положим

$$
n=m N=\sigma z \sqrt{N}
$$

Тогда, очевидно,

$$
\begin{aligned}
P_{N}(n) & =\frac{1}{2 \pi} \int_{-\pi}^{\pi} e^{-i t \sigma z \sqrt{N}} e^{-i t m N} \varphi^{N}(t) d t \\
& =\frac{1}{2 \pi} \int_{-\pi}^{\pi} e^{-i t \sigma z \sqrt{N}}(\bar{\varphi}(t))^{N} d t
\end{aligned}
$$

Сделав замену $x=\sigma t \sqrt{N}$, получаем, что

$$
P_{N}(n)=\frac{1}{2 \pi \sigma \sqrt{N}} \int_{-\pi \sigma \sqrt{N}}^{\pi \sigma \sqrt{N}} e^{-i x z}(\bar{\varphi}(x /(\sigma \sqrt{N})))^{N} d x
$$

Поскольку

$$
\frac{1}{2 \pi} e^{-z^{2} / 2}=\frac{1}{2 \pi} \int_{-\infty}^{\infty} e^{-i x z-x^{2} / 2} d x
$$

разность

$$
R_{N}(n)=2 \pi\left(\sigma \sqrt{N} P_{N}(n)-\frac{1}{\sqrt{2 \pi}} e^{-z^{2} / 2}\right)
$$


можно представить в виде суммы четырех интегралов

$$
\begin{aligned}
& I_{1}=\int_{-A}^{A}\left(((\bar{\varphi}(x) /(\sigma \sqrt{N})))^{N}-e^{-x^{2} / 2}\right) d x, \\
& I_{2}=-\int_{|x| \geqslant A} e^{-i x z-x^{2} / 2} d x, \\
& I_{3}=-\int_{A \leqslant|x| \leqslant \pi \sigma \sqrt{N}} e^{-i x z}\left(\bar{\varphi}(x /(\sigma \sqrt{N}))^{N} d x,\right. \\
& I_{4}=-\int_{\varepsilon \sigma \sqrt{N} \leqslant|x| \leqslant \pi \sigma \sqrt{N}} e^{-i x z}\left(\bar{\varphi}(x /(\sigma \sqrt{N}))^{N} d x,\right.
\end{aligned}
$$

где положительные постоянные $A$ и $\varepsilon$ будут выбраны позднее.

Покажем, что выбором $A, \varepsilon$ и $N$ величины $R_{N}(n)$ может быть сделана сколь угодно малой, точнее, покажем, что для произвольного $\delta>0$ выбором $A, \varepsilon$ и $N$ можно добиться выполнения неравенства $\left|R_{N}(n)\right| \leqslant \delta$.

Ясно, что

$$
\left|I_{2}\right| \leqslant \int_{|x| \geqslant A} e^{-x^{2} / 2} d x,
$$

и выбором достаточно большого $A$ можно добиться выполнения неравенства

$$
\left|I_{2}\right| \leqslant \frac{\delta}{4}
$$

Оценим интеграл

$$
I_{3}=-\int_{A \leqslant|x| \leqslant \pi \sigma \sqrt{N}} e^{-i x z}\left(\bar{\varphi}(x /(\sigma \sqrt{N}))^{N} d x .\right.
$$

Воспользуемся разложением характеристической функции

$$
\bar{\varphi}(t)=\frac{B\left(\theta e^{i t}\right)}{B(\theta)} e^{-i t m}
$$

Введем обозначение $a=e^{i t}-1$. Аналогичное (7) разложение имеет вид

$$
\ln B(\theta+a \theta)=\ln \left(B(\theta)+a \theta B^{\prime}(\theta)+\frac{1}{2}(a \theta)^{2} B^{\prime \prime}(\theta)+R_{r},\right.
$$

где $R_{2}=0\left(a^{3} \theta^{3}\right), R_{r}=0\left(a_{3} \theta^{r}\right)$ при $r \geqslant 3$. Далее,

$$
\begin{aligned}
\ln B(\theta+a \theta) & =\ln B(\theta)+\ln \left(1+\frac{a \theta B^{\prime}(\theta)}{B(\theta)}+\frac{(a \theta)^{2} B^{\prime \prime}(\theta)}{2 B(\theta)}+O\left(R_{r}\right)\right) \\
& =\ln B(\theta)+\frac{a \theta B^{\prime}(\theta)}{B(\theta)}+\frac{(a \theta)^{2} B^{\prime \prime}(\theta)}{2 B(\theta)}-\frac{(a \theta)^{2}\left(B^{\prime}(\theta)\right)^{2}}{2(B(\theta))^{2}}+O\left(R_{r}\right) .
\end{aligned}
$$

Ясно, что

$$
\begin{gathered}
a=e^{i t}-1=i t-\frac{t^{2}}{2}+O\left(t^{3}\right), \\
a m-i t m=-\frac{t^{2} m}{2}+O\left(m t^{3}\right) .
\end{gathered}
$$


Отсюда

$$
\begin{aligned}
\ln \bar{\varphi}(t) & =\ln \left(\frac{B\left(\theta e^{i t}\right)}{B(\theta)} e^{-i t m}\right) \\
& =a m+\frac{(a \theta)^{2} B^{\prime \prime}(\theta)}{2 B(\theta)}-\frac{(a \theta)^{2}\left(B^{\prime}(\theta)\right)^{2}}{2(B(\theta))^{2}}-i t m+O\left(R_{r}\right) \\
& =-\frac{t^{2}}{2}\left(\mathbf{M} \xi+\mathbf{M} \xi(x i-1)-(\mathbf{M} \xi)^{2}\right)+O\left(R_{r}\right)+O\left(m t^{3}\right) \\
& =-\frac{t^{2} \sigma^{2}}{2}+O\left(R_{r}\right)+O\left(m t^{3}\right) .
\end{aligned}
$$

Учитывая асимптотику $m$ и $\sigma$ (см. (8)), получаем, что

$$
\ln \bar{\varphi}(t)=-\frac{t^{2} \sigma^{2}}{2}+O\left(t^{3} \theta^{r}\right)
$$

откуда

$$
\bar{\varphi}(t)=e^{-t^{2} \sigma^{2} / 2+O\left(t^{3} \theta^{r}\right)},
$$

так что при $|t| \leqslant \varepsilon$ и достаточно малом $\varepsilon>0$ справедливо неравенство

$$
|\bar{\varphi}(t)| \leqslant e^{-t^{2} \sigma^{2} / 4}
$$

Оценивая теперь интеграл $I_{3}$, находим, что

$$
\begin{aligned}
\left|I_{3}\right| & \leqslant \int_{A \leqslant|x| \leqslant \varepsilon \sigma \sqrt{N}} \mid \bar{\varphi}\left(x /\left.(\sigma \sqrt{N})\right|^{N} d x \leqslant \int_{A \leqslant|x| \leqslant \varepsilon \sigma \sqrt{N}}\left(e^{-x^{2} /(4 N)}\right)^{N} d x\right. \\
& \leqslant \int_{A \leqslant|x|} e^{-x^{2} / 4} d x
\end{aligned}
$$

и выбором достаточно большого $A$ можно добиться выполнения неравенства

$$
\left|I_{3}\right| \leqslant \frac{\delta}{4}
$$

Итак, выбирая достаточно большое $A$ и достаточно малое $\varepsilon$, получаем, что для интегралов $I_{2}$ и $I_{3}$ выполняются неравенства $\left|I_{2}\right| \leqslant \delta / 4$ и $\left|I_{3}\right| \leqslant \delta / 4$.

При фиксированном $A$ интеграл $I_{1}$ может быть сделан сколь угодно малым выбором достаточно большого $N$, так как при $N \theta^{r} \rightarrow \infty$ справедлива теорема 3 о сходимости распределения случайной величины $S_{N}^{*}$ к стандартному нормальному распределению, так что при $N \rightarrow \infty$

$$
\bar{\varphi}(x /(\sigma \sqrt{N})) \rightarrow e^{-x^{2} / 2}
$$

равномерно в любом конечном интервале, например, при $|x| \leqslant A$. Выбирая достаточно большое $N$, получаем, что

$$
\left|I_{1}\right| \leqslant \frac{\delta}{4}
$$


При оценке интеграла $I_{4}$ приходится учитывать влияние значений $|\varphi(t)|$, возможно близких к единице в некоторых точках интервала $[-\pi, \pi]$, исключая, конечно, окрестность точки $t=0$, где уже оценен интеграл $I_{1}$. Такими точками являются точки $t_{k}^{(r)}=2 \pi k / r$, $|k|=0,1, \ldots,[r / 2]$. В этих точках сумма первых двух членов разложения характеристической функции $\varphi(t)$, равная

$$
\frac{1}{B(\theta)}\left(b_{0}+\frac{b_{r} \theta^{r}}{r !} e^{i t r}\right)
$$

имеет то же значение, что и при $t=0$.

Третий по величине член разложения, равный $b_{s} \theta^{s} e^{i t s} / s !$, принимает то же значение, что и в точке $t=0$, в точках $t_{k}^{(s)}=2 \pi k / s,|k|=0,1, \ldots,[s / 2]$.

Выделим $\varepsilon$-окрестности точек $t_{k}^{(r)},|k|=0,1, \ldots,[r / 2]$, и $t_{k}^{(s)},|k|=0,1, \ldots,[s / 2]$. Обозначим $R_{r, \varepsilon}$ часть интервала $[-\pi, \pi]$, полученную удалением $\varepsilon$-окрестностей точек $t_{k}^{(r)},|k|=0,1, \ldots,[r / 2]$, и $R_{s, \varepsilon}$ часть интервала $[-\pi, \pi]$, полученную удалением $\varepsilon$-окрестностей точек $t_{k}^{(s)},|k|=0,1, \ldots,[s / 2]$. По условию теоремы, максимальный шаг решетки, на которой лежат точки $0, r, s$, равен единице, поэтому наибольшее общее кратное чисел $r$ и $s$ равно 1. Отсюда следует, что, кроме точки 0 , все точки $t_{k}^{(r)}, t_{k}^{(s)}$ различны, и, значит, все удаленные $\varepsilon$-окрестности этих точек при достаточно малом $\varepsilon>0$ не пересекаются, за исключением окрестностей точки 0. Поэтому

$$
R_{r, \varepsilon} \cup R_{s, \varepsilon}=[-\pi,-\varepsilon] \cup[\varepsilon, \pi]
$$

Для оценки интеграла $I_{4}$ представим его в виде

$$
I_{4}=\sigma \sqrt{N} \int_{\varepsilon \leqslant|t| \leqslant \pi} e^{-i t z \sigma \sqrt{N}} \bar{\varphi}(t) d t
$$

Из этого представления следует, что

$$
\left|I_{4}\right| \leqslant \sigma \sqrt{N} \int_{\varepsilon \leqslant|t| \leqslant \pi}|\varphi(t)| d t .
$$

Оценим модуль характеристической функции $\varphi(t)$. Для краткости введем обозначения

$$
\begin{aligned}
a_{r} & =\frac{b_{r} \theta^{r}}{b_{0} r !}, & & a_{s}=\frac{b_{s} \theta^{s}}{b_{0} s !}, \\
\alpha_{r} & =r t, & \alpha_{s} & =s t .
\end{aligned}
$$


Нетрудно видеть, что

$$
\begin{aligned}
\varphi(t) & =\left(1+a_{r} e^{i \alpha_{r}}+a_{s} e^{i \alpha_{s}}+O\left(\theta^{s+1}\right)\right) /\left(1+a_{r}+a_{s}+O\left(\theta^{s+1}\right)\right) \\
& =\frac{1+a_{r} e^{i \alpha_{r}}+a_{s} e^{i \alpha_{s}}+O\left(\theta^{s+1}\right)}{\left(1+a_{r}\right)\left(1+a_{s} /\left(1+a_{r}\right)+O\left(\theta^{s+1}\right)\right.} \\
& =\frac{\left(1+a_{r} e^{i \alpha_{r}}\right) /\left(1+a_{r}\right)+a_{s} e^{i \alpha_{s}}+O\left(\theta^{s+1}\right)}{1+a_{s}+O\left(\theta^{s+1}\right)} \\
& =\left(\frac{1+a_{r} e^{i \alpha_{r}}}{1+a_{r}}+a_{s} e^{i \alpha_{s}}+O\left(\theta^{s+1}\right)\right)\left(1-a_{s}+O\left(\theta^{s+1}\right)\right) \\
& =\frac{1+a_{r} e^{i \alpha_{r}}}{1+a_{r}}+a_{s} e^{i \alpha_{s}}-\frac{a_{s}\left(1+a_{r} e^{i \alpha_{r}}\right)}{1+a_{r}}+O\left(\theta^{s+1}\right) \\
& =1-\frac{a_{r}}{1+a_{r}}\left(1-e^{i \alpha_{r}}\right)-a_{s}\left(1-e^{i \alpha_{s}}\right)+O\left(\theta^{s+1}\right) \\
& =1-\frac{a_{r}}{1+a_{r}}\left(1-\cos \alpha_{r}-i \sin \alpha_{r}\right)-a_{s}\left(1-\cos \alpha_{s}-i \sin \alpha_{s}\right)+O\left(\theta^{s+1}\right) .
\end{aligned}
$$

Переходя к оценке модуля характеристической функции, находим, что

$$
\begin{aligned}
|\varphi(t)|^{2}= & \left|1-\frac{a_{r}}{1+a_{r}}\left(1-\cos \alpha_{r}-i \sin \alpha_{r}\right)-a_{s}\left(1-\cos \alpha_{s}-i \sin \alpha_{s}\right)+O\left(\theta^{s+1}\right)\right|^{2} \\
= & \left(1-\frac{a_{r}}{1+a_{r}}\left(1-\cos \alpha_{r}-a_{s}\left(1-\cos \alpha_{s}\right)\right)^{2}\right. \\
& +\left(\frac{a_{r}}{1+a_{r}} \sin \alpha_{r}+a_{s} \sin \alpha_{s}\right)^{2}+O\left(\theta^{s+1}\right) \\
= & 1-\frac{2 a_{r}}{1+a_{r}}\left(1-\cos \alpha_{r}\right)+\frac{a_{r}^{2}}{\left(1+a_{r}\right)^{2}}\left(1-\cos \alpha_{r}\right)^{2}-2 a_{s}\left(1-\cos \alpha_{s}\right) \\
& +\frac{a_{r}^{2}}{\left(1+a_{r}\right)^{2}} \sin ^{2} \alpha_{r}+O\left(\theta^{s+1}\right) \\
=1 & -\frac{2 a_{r}}{1+a_{r}}\left(1-\cos \alpha_{r}\right)+\frac{a_{r}^{2}}{\left(1+a_{r}\right)^{2}}\left(1-2 \cos \alpha_{r}+\cos ^{2} \alpha_{r}+\sin ^{2} \alpha_{r}\right) \\
& -2 a_{s}\left(1-\cos \alpha_{s}\right)+O\left(\theta^{s+1}\right) \\
= & 1-\frac{2 a_{r}}{1+a_{r}}\left(1-\cos \alpha_{r}\right)+\frac{2 a_{r}^{2}}{\left(1+a_{r}\right)^{2}}\left(1-\cos \alpha_{r}\right) \\
& -2 a_{s}\left(1-\cos \alpha_{s}\right)+O\left(\theta^{s+1}\right)
\end{aligned}
$$

Отсюда следует, что

$$
|\varphi(t)|^{2} \leqslant 1-a_{r}\left(1-\cos \alpha_{r}\right)-2 a_{s}\left(1-\cos \alpha_{s}\right)+O\left(\theta^{s+1}\right) .
$$

Если $t \in R_{r, \varepsilon}$, то есть $t$ не лежит в $\varepsilon$-окрестности ни одной из точек $t_{k}^{(r)}$, $|k|=1,2, \ldots,[r / 2]$, то для любой из этих точек $\left|t_{k}^{(r)}-t\right| \varepsilon$, а значит, $|2 \pi k / r-t| \geqslant \varepsilon$ и $\left|2 \pi k-\alpha_{r}\right| \geqslant \varepsilon r$. Поэтому

$$
1-\cos \alpha_{r} \geqslant 1-\cos \varepsilon r \geqslant 2 c,
$$


где $c$ - положительная постоянная. Из (9) следует, что

$$
|\varphi(t)|^{2} \leqslant 1-a_{r}\left(1-\cos \alpha_{r}\right)+O\left(\theta^{s+1}\right),
$$

поэтому в силу того, что $a_{r}=O\left(\theta^{r}\right)$, из (10) следует, что

$$
\begin{aligned}
|\varphi(t)|^{2} & \leqslant 1-2 c a_{r}+O\left(\theta^{s+1}\right) \\
& \leqslant 1-c a_{r} \leqslant e^{2 b \theta^{r}},
\end{aligned}
$$

где $b$ - положительная постоянная и $t \in R_{r, \varepsilon}$.

Итак,

$$
|\varphi(t)| \leqslant e^{-b \theta^{r}},
$$

и для интеграла $\sigma \sqrt{n} \int_{R_{r, \varepsilon}}|\varphi(t)|^{N} d t$ с учетом того, что $\sigma^{2}=O\left(\theta^{r}\right)$, справедлива оценка

$$
\begin{aligned}
\sigma \sqrt{N} \int_{R_{r, \varepsilon}}|\varphi(t)|^{N} d t & \leqslant c_{1} \sqrt{\theta^{r} N} \int_{-\pi}^{\pi} e^{-b \theta^{r} N} d t \\
& =2 \pi c_{1} \sqrt{\theta^{r} N} e^{-b \theta^{r} N},
\end{aligned}
$$

где $c_{1}$ - положительная постоянная.

Таким образом, эта часть интеграла $I_{4}$ стремится к нулю в условиях теоремы.

Пусть теперь $t \notin R_{r, \varepsilon}$, то есть $t$ лежит в $\varepsilon$-окрестности одной из точек $t_{k}^{(r)}$, $|k|=1,2, \ldots,[r / 2]$, так что существует $k_{0}$ такое, что $\left|t_{k_{0}}^{(r)}-t\right| \leqslant \varepsilon$, откуда следует, что

$$
\left|r t_{k_{0}}^{(r)}-\alpha_{r}\right| \leqslant r \varepsilon
$$

и значит, $\alpha_{r}=2 \pi k_{0}+r \tau$, где $|\tau| \leqslant \varepsilon$. При достаточно малом $\varepsilon>0$,

$$
1-\cos \alpha_{r}=1-\cos r \tau \geqslant c_{2} \tau^{2}
$$

Если $t$ лежит в $\varepsilon$-окрестности точки $t_{\left.k_{(}\right)}^{(r)}$, то $t \in R_{s, \varepsilon}$, и поэтому

$$
1-\cos \alpha_{s} \geqslant 1-\cos \varepsilon s \geqslant 2 c_{3},
$$

где $c_{3}$ - положительная постоянная.

Теперь из (9), (11) и (12) следует, что в каждой из $\varepsilon$-окрестностей любой из точек $t_{k}^{(r)}$, $|k|=1,2, \ldots,[r / 2]$,

$$
\begin{aligned}
|\varphi(t)|^{2} & \leqslant 1-c_{2} a_{r} \tau^{2}-2 c_{3} a_{s}+O\left(\theta^{s+1}\right) \\
& \leqslant 1-c_{2} a_{r} \tau^{2}-c_{3} a_{s} \\
& \leqslant 1-c_{4} \theta^{r} \tau^{2}-c_{5} \theta^{s}
\end{aligned}
$$

где $c_{4}, c_{5}-$ положительные постоянные и $\tau \leqslant \varepsilon$. 
Оценивая теперь часть интеграла $I_{4}$ по $\varepsilon$-окрестностям всех точек $t_{k}^{(r)}$, $|k|=1,2, \ldots,[r / 2]$, находим, что

$$
\begin{aligned}
\sigma \sqrt{N} \int_{[-\pi, \varepsilon] \cup[\varepsilon, \pi] \backslash R_{r, \varepsilon}}|\varphi(t)|^{N} d t & \leqslant c_{6} \sqrt{\theta^{r} N} r \int_{-\varepsilon}^{\varepsilon} e^{-c_{4} \theta^{r} N \tau^{2}-c_{5} \theta^{s} N} d \tau \\
& \leqslant c_{6} r e^{-c_{5} \theta^{s} N} \int_{-\infty}^{\infty} e^{-c_{4} u^{2}} d u \leqslant c_{7} e^{-c_{5} \theta^{s} N},
\end{aligned}
$$

где последний интеграл получен заменой переменной $u=\sqrt{\theta^{r} N} \tau$ и расширением области интегрирования. Правая часть этой цепочки неравенств при $N \rightarrow \infty$ и $\theta \rightarrow 0$ стремится к нулю, так как по условию теоремы $\theta^{s} N \rightarrow \infty$.

Итак, обе части интеграла $I_{4}$, по областям $R_{r, \varepsilon}$ и $[-\pi,-\varepsilon] \cup[\varepsilon, \pi] \backslash R_{r, \varepsilon}$, стремятся к нулю, и выбором достаточно большого $N$ можно добиться выполнения неравенства

$$
\left|I_{4}\right| \leqslant \frac{\delta}{4}
$$

Таким образом, доказательство теоремы 4 завершено.

Итак, если $N \theta^{s} \rightarrow \infty$ при $N \rightarrow \infty$, то для $S_{N}$ справедлива локальная предельная теорема (теорема 4) о сходимости к нормальному распределению на решетке целых чисел. Если же $N \theta^{r} \rightarrow \lambda r ! b_{0} / b_{r}, \lambda>0$, то для $S_{N}$ справедлива теорема 2 о сходимости к распределению Пуассона с параметром $\lambda$ на решетке целых неотрицательных чисел с шагом $r$. Таким образом, при промежуточных порядках стремления параметра $\theta$ к нулю происходит переход распределения $S_{N}$ с решетки всех целых чисел на решетку неотрицательных целых чисел с шагом $r$. Этот переход будет описан в другой статье, так как для изучения деталей этого перехода требуются другие методы исследования.

\section{Список литературы}

1. Гнеденко Б. В., Колмогоров А. Н., Предельные распределения для сумм независимых случайных величин. ГИТТЛ, Москва-Ленинград, 1949.

2. Казимиров Н. И., Павлов Ю. Л., Одно замечание о лесах Гальтона-Ватсона. Дискретная математика (2000) 12, №1, 47-59.

3. Казимиров Н. И., О некоторых условиях отсутствия гигантской компоненты в обобщенной схеме размещения. Дискретная математика (2002) 14, №2, 107-118.

4. Kolchin A. V., On the number of hyperforests. J. Math. Sci. (1995) 76, 2250-2258.

5. Kolchin A. V., On the asymptotic behaviour of the number of hyperforests. In: Probabilistic Methods in Discrete Mathematics. VSP, Utrecht, 1996, pp. 285-294.

6. Колчин А. В., Предельные теоремы для обобщенной схемы размещения. Дискретная математика (2003) 15, №4, 148-157.

7. Колчин В. Ф., Один класс предельных теорем для условных распределений. Литовский матем. сборник (1968) 8, №1, 111-126.

8. Колчин В. Ф., Севастьянов Б. А., Чистяков В. П., Случайные размещения. Наука, Москва, 1976.

9. Колчин В. Ф., Случайные отображения. Наука, Москва, 1984.

10. Колчин В. Ф., Случайные графы. Физматлит, Москва, 2000.

11. Колчин В. Ф., Асииптотические методы теории вероятностей. МИЭМ, Москва, 1984. 
12. Мухин А. Б., Локальные предельные теоремы для решетчатых случайных величин. Теория вероятностей и ее применения (1991) 36, №4, 660-674.

13. Myllari T. B., Limit distributions of the number of leaves of a Galton-Watson forest. In: Probabilistic Methods in Discrete Mathematics. VSP, Utrecht, 2002, pp. 257-271.

14. Pavlov Yu. L., Random forests. VSP, Utrecht, 2000.

15. Павлов Ю. Л., Предельные теоремы для объемов деревьев непомеченного графа случайного отображения. Дискретная математика (2004) 16, №3, 63-75.

16. Павлов Ю. Л., Предельные теоремы для объемов деревьев в случайном непомеченном лесе. Дискретная математика (2005) 17, №2, 70-86.

17. Тимашёв А. Н., Об асимптотических разложениях в локальных предельных теоремах в равновероятных схемах размещения частиц по различным ячейкам. Дискретная математика (2000) 12, №1, 60-69.

18. Тимашёв А. Н., О распределении числа циклов заданной длины в классе подстановок с известным числом циклов. Дискретная математика (2001) 13, №4, 60-72.

19. Тимашёв А. Н., Случайные свободные деревья и леса с ограничениями на кратности вершин. Дискретная математика (2004) 16, №4, 117-133.

20. Тимашёв А. Н., Большие уклонения для числа деревьев заданного объема и максимального объема дерева в случайном лесе. Дискретная математика (2006) 18, №3, 77-84.

Статья поступила 8.10.2006. 